\title{
AN AGENT-BASED APPROACH TO TRANSPORT CHAIN MANAGEMENT
}

\author{
Paul Davidsson, Jan A. Persson \\ Blekinge Institute of Technology, Sweden \\ paul.davidsson@bth.se,jan.persson@bth.se \\ Andreas Jacobsson \\ School of Technology, Malmö University, Sweden \\ andreas.jacobsson@mah.se
}

\begin{abstract}
Keywords: Dynamic e-business collaboration, Virtual organizations, Collaborative business systems, Supply chain management.

Abstract: A novel application of agent-mediated electronic commerce is presented. It concerns developing and maintaining efficient and effective transport solutions. The suggested approach is inspired by the concepts of virtual enterprises and breeding environments, as well as peer-to-peer technology. We discuss the requirements of such an approach and outline a software architecture meeting these requirements.
\end{abstract}

\section{INTRODUCTION}

It is well known that collaboration is essential for improved business processes and increased profitability within supply chains in the e-commerce area. Consequently, there is a need for increased collaboration between transport chain actors, e.g. transport users, transport coordinators, transport operators and terminal operators, in order to achieve efficient, cost-effective, and agile co-modal transport chains. The proposed approach Plug and Play Transport Chain Management (PnP TCM) focus on businessto-business (B2B) interaction for developing transport solutions, activity coordination, and business transactions, based on the FREIGHTWISE framework (FWF) outlined by Fjørtoft et al. (2008). (FREIGHTWISE (Management Framework for Intelligent Intermodal Transport) is a EU-funded FP6 Integrated Project with 55 partners from 14 different countries. Its goal is to enable modal shift of cargo flows from road to intermodal transport through improved information exchange between all stakeholders across all transport modes) The PnP TCM approach is inspired by the more general Plug and Play Business concept as introduced by Davidsson et al. (2006), Jacobsson et al. (2006), and Jacobsson et al. (2008).

The PnP TCM approach aims to:
- increase integration of transport chains by making information about transport services easily accessible (partially by providing interoperability between the ERP systems of the involved actors),

- increase adaptivity and agility of transport chains by providing a match-making functionality that makes it easier for potential transport chain actors to find each other and negotiate over terms of collaboration with potential partners, and

- lower the entry barriers for small-sized companies to participate in highly integrated transport chains by providing low cost open source and easy-to-use software tools.

Moreover, it should ideally be implemented in a completely distributed fashion, both with respect to control and information, and it should be seamlessly interoperable with relevant legacy ERP systems of the participating actors. Figure 1 illustrates the PnP TCM approach where each actor use the FWF (or PnP TCM) software to set up and participate in a transport chain. The FWF software has two types of interfaces, one type interacts directly with legacy ERP (Enterprise Resource Planning) systems, or other relevant information systems, and the other is a simple web interface, which may be particularly useful for small enterprises. 


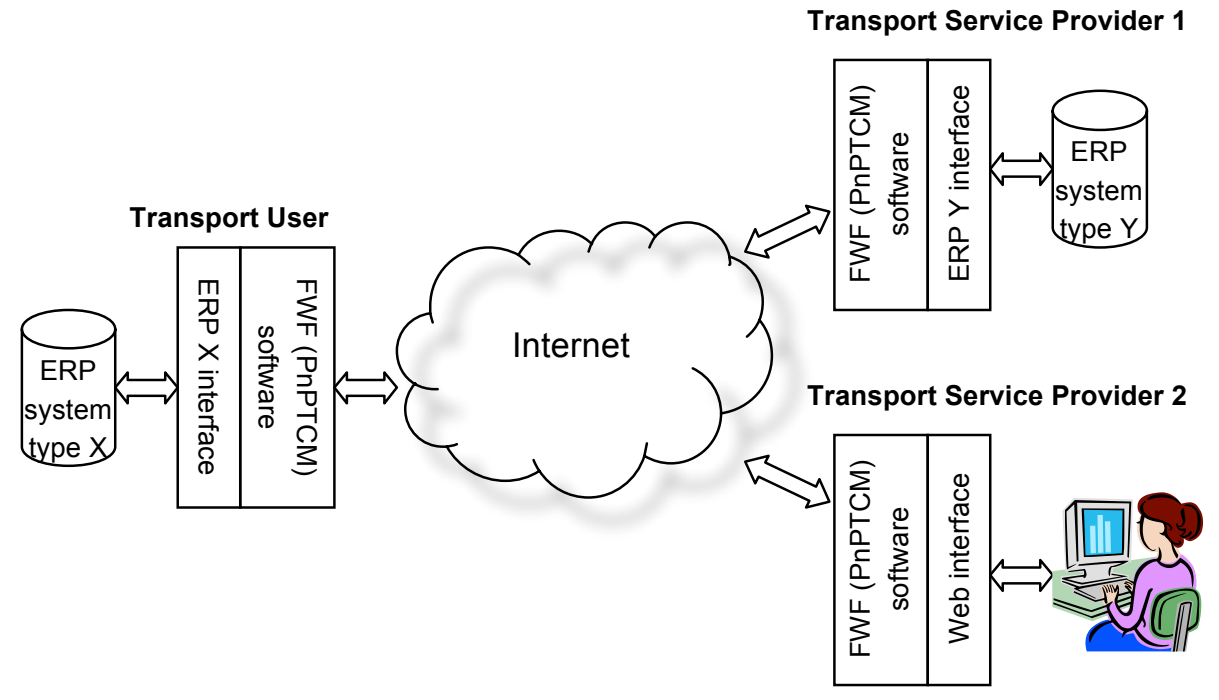

Fig. 1. An illustration of the PnP TCM approach with three participating transport chain actors.

There are four roles identified in the FWF. The Transport User is anyone that needs to have cargo transported. The Transport Service Provider is the role that ensures the transport of the cargo including the management of the transport services and the operation of the transport means and handling equipment. The Traffic Manager is responsible for information regarding the infrastructure (static or dynamic) related to planning and executing transport. Finally, the Transport Regulator monitors that all transport services are completed according to existing rules and regulations.

To achieve interoperable and cost-efficient collaboration within transport chain management, these roles are important when identifying the functional requirements of the PnP TCM software. In the next section, we will see how this can be achieved.

\section{FUNCTIONAL SPECIFICATION}

As indicated in Figure 1, we here focus on two roles of the FWF, the Transport User and the Transport Service Provider. Moreover, we concentrate on the Planning phase as the two other phases, Execution and Completion, concern more straightforward interaction between the involved actors. A more detailed illustration of the interaction between Transport Users and Transport Service Providers during the Planning process is provided in Figure 2.
At the beginning of the planning process, the Transport User specifies its transport demands, resulting in an initial Transport Execution Plan (TEP) covering the complete transport from origin to destination. The TEP is initial, or preliminary in the sense that it specifies the items that are to be transported, the desired time and date of collection and delivery, the origin and destination, and the condition of the items during the transport (e.g., ambient temperature conditions), but not which Transport Service(s) to use. The next step is to find relevant transport services that potentially can meet the transport demands specified in the initial TEP. This is done by searching among the Transport Service Descriptions (TSD) published by the Transport Service Providers and by selecting those that (at least partially) match the TEP.

This set of TSDs then provides the input to the next step, which is to find the sequence of TSDs that satisfies the requirements of the TEP, and, if more than one such sequence is found, the one that is "best" according to one or more criteria, such as, cost, reliability, environmental impact, etc. If no sequence that satisfies the requirements is found, the TEP needs to be revised, e.g. with respect to desired time of delivery. When a sequence is found, it is time to negotiate the detailed conditions for each of the TSD with the corresponding Transport Service Provider. This is done in terms of a TEP corresponding to that part of the transport, i.e., for 


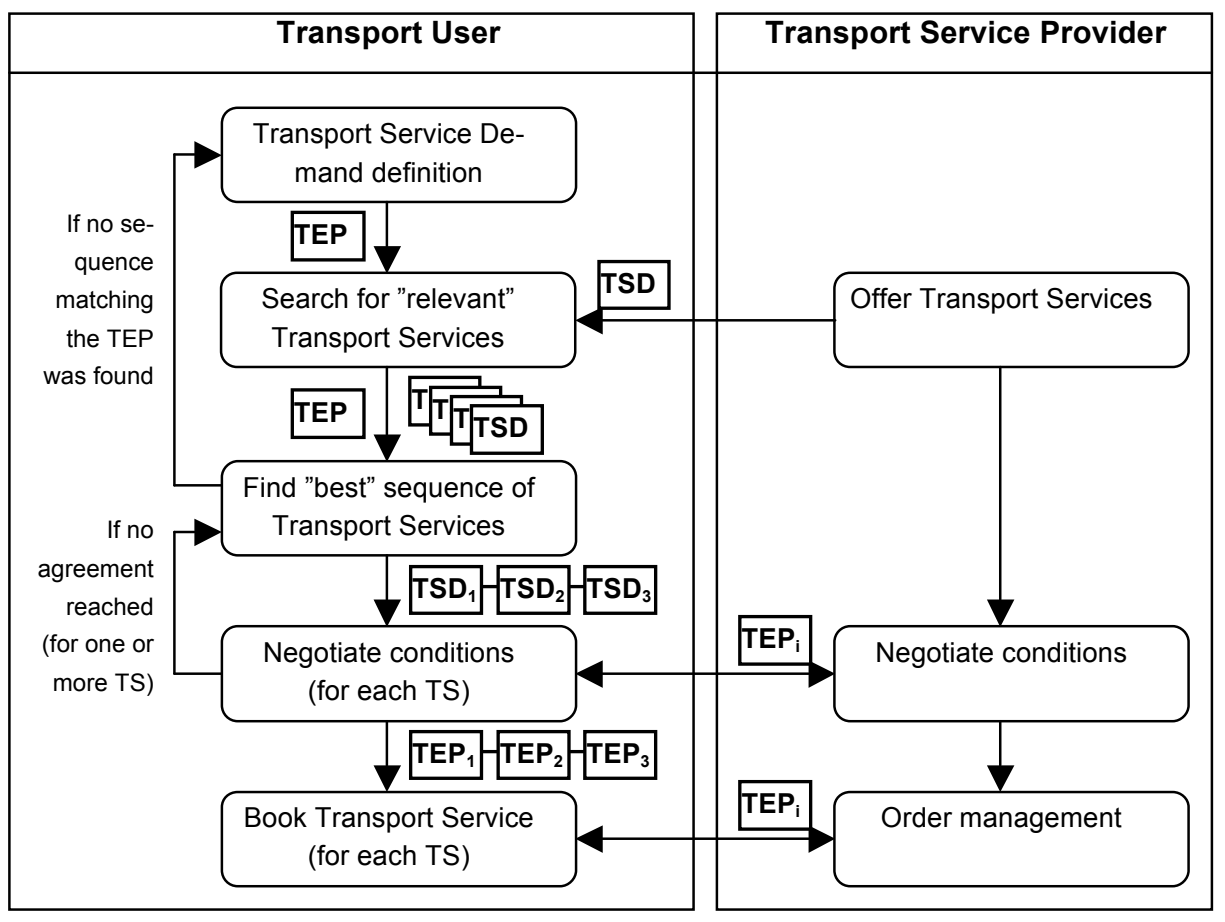

Fig. 2. The interaction between Transport Users and Transport Service Providers during the Planning phase.

each TSD in the sequence, a TEP will be produced. And the sequence of TEPs will correspond to the initial TEP covering the complete transport from origin to destination. If no agreement is reached for one (or more) of the TSDs, it (they) will be removed from the set of potential TSDs and the finding of the best sequence will be resumed. The final step of the planning phase is to book the transport services (one for each TEP in the sequence).

\section{REALIZATION}

Due to the large number of actors involved and their different roles, we believe that there is a need for a new type of software for supporting the FWF, building on concepts, such as, Internet communities, and Virtual Enterprises, and technologies, such as, Peerto-Peer software, and Software Agents.

\subsection{Conceptual View}

In PnP TCM, we view transport chains as virtual enterprises, which may be defined as temporary alliances of enterprises that come together to share skills or core competencies and resources in order to better respond to business opportunities, and whose cooperation is supported by computer networks (Camarinha-Matos and Afsarmanesh, 2003). The common business goal for the participants of a transport chain is to deliver the goods to the final destination in time. Another important concept for PnP TCM is Internet community. Potential transport chain actors dynamically join a PnP TCM community by (installing and) executing the agent-based PnP TCM software. To enter the community, an actor needs to declare its address and other formalities, as well as agreeing on an end-user license agreement. A Transport Service Provider needs, in addition, to provide a Transport Service Description (TSD) for each transport service it provides. The TSDs should be updated continuously so as to mirror the current situation. The community is dynamic in the sense that enterprises may (in principle) join and leave the community at any time. The PnP TCM community can be seen as a breeding environment (Camarinha-Matos and Afsarmanesh, 2003) for transport chains. A breeding environment represents an association of enterprises that have both the potential and the ambition to collaborate with each other through an interoperable infrastructure. A realized transport solution within the breeding environment is an agent-supported virtual enterprise. 
The set of installed PnP TCM software agents in a community can be viewed to form an artificial society. Davidsson (2001) lists four types of artificial society structures: open, closed, semi-closed, and semi-open artificial societies. The four categories balance the trade-off between important society properties, such as, openness, flexibility, stability, and trustfulness differently. In open societies there are no restrictions at all for joining the society. In a FWF setting, this may correspond to that all interaction is performed in an ad-hoc fashion, e.g., TSDs are published openly on the WWW and the Transport Users need to find the offers through the use of general WWW search engines, etc. An open society supports openness and flexibility, but not stability and trustfulness, and the opposite is true for closed societies. In a FWF setting, a closed society solution may be a completely centralized system in which all information is stored and through which all interaction between the transport chain actors is mediated. In many situations, such as in breeding environments for transport chains, there is a need for societies that better balance the trade-off between the society properties. We will therefore limit our discussion to the two intermediate categories.

An important actor in the context of artificial societies is the "owner" of the society, or environment owner. By this we mean, the person or organization that have the power to decide which software entities may enter the society, which roles they are allowed to occupy, what communication language should be used, the set of norms and rules that are valid within the society, etc.

In semi-closed artificial societies, external software agents are not allowed to enter. However, actors have the possibility to initiate new software agents in the society, which will act on behalf of the actor. In semi-closed societies, there is a (central) physical environment, in which the agents (representing their owners) execute and communicate with other agents. This requires that the actors' agents can access some level of mutual communication properties, which are included in the breeding environment. Semi-closed societies convey almost the same degree of openness as semi-open societies, but are less flexible. From a FWF perspective, they fail to meet the requirement of a distributed solution. On the other hand, they have a larger potential for implementing important society attributes, such as, security and trustfulness.

The main difference to semi-closed artificial societies is that, in semi-open societies, agents execute locally on the clients individual computer systems. However, another distinction is that the environment owner is no longer in control of the agents even though the environment owner still has the power to, for instance, dictate the rules of engage- ment within the society. In order to meet securityrelated requirements, semi-open societies are equipped with a gate-keeper, to which every agent needs to connect before entering the society.

When analyzing semi-open societies, it is useful to make a distinction between two types of such societies, those with a centralized and those with decentralized communication architecture. In the case of centralized communication, the agents run on the members' own individual computer systems, but all communication between the agents is routed via a central server. In decentralized communication, the agents run on the members' individual computer systems and all communication is conducted directly between these end nodes. As the vision of FWF consists of a distributed solution, a semi-open agent society with decentralized communication seems desirable.

\subsection{PnP TCM Software Requirements}

The concept of PnP TCM relies on an integrated set of agent-based ICT-tools that support the creation and management of transport chains. ICTinfrastructures for business creation and collaboration are particularly relevant to small-sized transport chain actors. While allowing themselves to maintain their business independence, they are able to reach otherwise unreachable markets and to take advantage of economies of scale with the support of ICT. Thus, the realization of ICT-based collaboration support is envisioned to boost transport chain management.

A PnP TCM community requires certain general functionalities. One example is the gate-keeper facility that regulates the entering (and leaving) of the transport chain actors (and the software agents acting on their behalf) and registers them as members of the community. Moreover, a surveillance mechanism that monitors the behavior of members may be necessary in order to cope with malicious acts (Davidsson et al., 2009). In addition, the gatekeeper facility, being a trusted third party, may also mediate the payment of transport services, contract validation, etc. In the planning phase, there are a number of functions that are helpful for the Transport User in forming a successful transport chain:

- finding potential transport services to be included in the transport chain from a possibly huge number of offered services distributed through the WWW,

- finding the best path of transport services, according to some criteria, from origin to destination out of the set of potential transport services, 
- $\quad$ supporting the negotiations with Transport Service Providers,

- $\quad$ supporting contract design, and

- $\quad$ supporting the transport booking.

When the planning phase is finished and a transport chain (virtual enterprise) is formed, the PnP TCM software should provide support also for the execution phase, i.e., the management of the actual activities within the transport chain. This support may be on a quite shallow level, e.g., transactions of information between actors, but may also support and facilitate more complex coordination and synchronization of activities.

As stated by Fjørtoft et al. (2008), only a limited number of information types, or packages, need to be supported. In addition to TSD and TEP, Transport Execution Status and Transport Item Status need to be exchanged between Transport User and Transport Service Provider during the execution phase. In the interaction with Traffic Managers there are two additional packages that should be handled, Transport Operation Status, as well as Network and Traffic Status. These information packages need to be transferred by the software agents in an efficient and secure way in order to reduce the administrational costs of the actors, as well as reducing the risk of inaccuracy in information. In promoting security, the gate-keeper functionality, with its guarding and surveillance features, come well at hand.

In addition to the functional requirements presented above, there are some relevant non-functional requirements that should be met, which could be specified in terms of quality attributes (and measured using some metric). Based on interviews with transport chain actors, we believe that the following quality attributes are important for PnP TCM software: scalability, flexibility, performance, cost, usability, and security (which is represented by the requirements of confidentiality, integrity, availability).

\subsection{Architecture}

The choice of system architecture is closely related to the system's performance in terms of a number of the criteria listed above. An extreme system architecture is a traditional client-server architecture where all information, e.g., the Transport Service Descriptions, is stored on a central server and all communication is routed through this server. The other extreme is a completely distributed architecture where no information is stored centrally. In order to implement a semi-open society, however, a hybrid architecture seems necessary. For instance, the gate-keeper facility is difficult to realize in a strictly distributed manner as it assumes some authorizing organization.

Compared to a centralized architecture, a distributed architecture supports many of the quality attributes, e.g., flexibility, scalability, and dynamicity. Also, the risk of single point of failure may be avoided increasing the robustness of the system. A decentralized paradigm, for instance, built on Peerto-Peer (P2P) technology (Oram, 2001) may be preferable for the PnP TCM software, because no central authority determines how the participants interact or coordinates them in order to accomplish some task, and minimal use of central repositories of information is needed. A pure $\mathrm{P} 2 \mathrm{P}$ system does not make use of the notions of clients and servers; instead it uses the notion of peers, which function both as clients and servers simultaneously. Moreover, there is no central server or router managing the communication. In addition, there are hybrid P2P systems that have some central server functions, such as, keeping (and providing) information about peers in the network.

It is possible to distinguish between structured and unstructured P2P systems. A structured P2P system uses a globally consistent protocol for finding relevant peers, using, e.g. distributed hash tables like the Chord Protocol (Stoica et al., 2001). An unstructured P2P system, based on, e.g. Gnutella (an open protocol used by many $\mathrm{P} 2 \mathrm{P}$ systems), a query needs to be "flooded" through the network of peers. Although many of the popular P2P systems are unstructured, this flooding causes a large amount of signaling traffic and therefore typically shows poor search efficiency, and sometimes the query even may not be resolved. Thus, to implement the search for Transport Service Providers offering relevant transport services, it seems as if a structured approach should be chosen. It is also possible to make use of a slightly more centralized approach, where the gate-keeper handles abstract (high level) descriptions of the transport services that a Transport Service Provider offers, for instance, "ship line between port A and port B", "truck transports in area C", "train transport in country D", etc. It is assumed that these high level descriptions do not change often. When they are changed, the Transport Service Provider (through the PnP TCM software) may notify the gate-keeper, who then broadcasts this information to the Transport User PnP TCM clients. This would make the search for relevant transport services more efficient, at the expense of some overhead communication. 


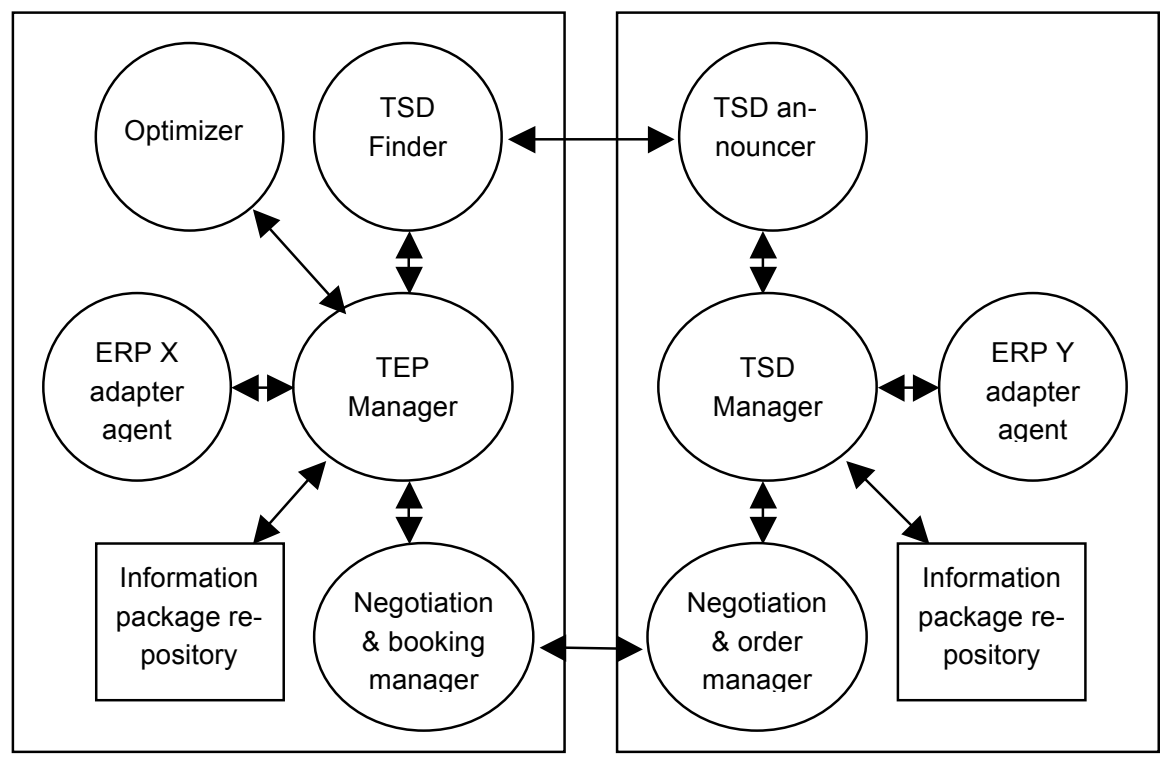

Fig. 3. Possible architectures of PnP TCM software clients (with adapter agent interfaces).

A P2P infrastructure self-configures and nodes can coordinate autonomously in order to search for resources, find them, and interact together. P2P being a paradigm that allows building dynamic overlay networks, it can be used in order to realize an environment that manages a dynamic network of business relations. Dealing with business-sensitive assets, searching and retrieval of contents should be made secure and trustable. This can be implemented using, for instance, the gate-keeper facility and the environment owner, which can govern interaction by means of rules and regulation. The $\mathrm{P} 2 \mathrm{P}$ infrastructure provides an environment in which every organization can make its knowledge and services available to other organizations while keeping complete control over them. Each organization can autonomously manage this task without having to delegate it to an external central authority that could be perceived as less trusted than the organization itself, and should be the object of an external (to the collaborating network) agreement between all the involved organizations.

An important part of the PnP TCM software is to find the best sequence of transport services. A large number of optimization algorithms have been developed that can be applied to solve this task, e.g., based on Integer Programming (Wolsey, 1998).

From the users' perspective, the complexity of the process of setting up the transport chain should be hidden by the PnP TCM software. There are thus, at least, two types of interfaces:

1. A web-based interface, which can be used by all types of users, independently of company size and IT maturity. One version for the Transport User, which consists of a number of different views specialized for each of the phases in the process, and one version for the Transport Service Provider, also with a number of different views.

2. An adapter agent interface, which makes the PnP TCM software interoperable with the user's ERP or other legacy system. An adapter may have to be developed for each ERP system, but once it is developed it can be reused by other organizations using that ERP system. One approach to implement the adapters is the general wrapper agent solution based on open source freeware introduced by Davidsson et al. (2005) that makes it possible for any business system to exchange (administrational) information with any other business system.

To summarize, the different agents of the PnP TCM software and how they interact are illustrated in Figure 3. These agents have different degrees of autonomy, from the quite reactive TSD announcer agent to the quite sophisticated manager agents. 


\section{RELATED WORK}

With respect to finding agreements through negotiation, there is a long tradition in the area of agentbased systems of studying this topic, for instance using the Contract Net Protocol (Smith, 1980) and computational auctions (Rosenschein and Zlotkin, 1994). Within the negotiation area (cf. Jennings et al. (2001)), four different components are relevant for the PnP TCM setting, namely: a negotiation set, which represents the space of possible obligations that agents can make, a protocol, which defines the legal obligations that the agents can make, a collection of strategies, one for each agent, which determines what obligations the enterprises will make, and a rule that determines when the negotiation is over and the deal has been closed. Negotiation results in an electronic contract, which govern the collaboration process. Electronic contracts are to be regarded as virtual representations of traditional contracts, i.e., "formalizations of the behavior of a group of agents that jointly agree on a specific business activity" (Cardoso and Oliveira, 2005). Electronic contracts usually have a set of identified roles, obligations or prohibitions to be fulfilled by the parties involved in the relation. PnP TCM software focuses on obligations, i.e., that an agent's role is defined by the obligation, which it has towards another agent to bring about a certain state of affairs before a certain deadline.

Several examinations on current state of the art technologies useful for building ICT-infrastructures with the purpose of collaboration within virtual enterprises have been undertaken (cf. CamarinhaMatos and Afsarmanesh (2003)). Some common conclusions are that multi agent technology constitutes a promising contributor to the development of support infrastructures and services. Internet and web technologies, such as web services, represent a fast growing sector with large potential in interenterprise collaboration support. However, further support in terms of supporting multi-lateral collaboration is necessary. A number of emerging technologies, e.g., service-oriented architectures, enterprise application integration, the semantic web, and countless collections of software standards (cf. the ebXML framework) are likely to provide important contributions. As a solution, it seems that Microsoft's BizTalk Server, and similar solutions, are the most sophisticated alternative for interenterprise collaboration widely available. However, being a centralized proprietary client-server solution it has several disadvantages, such as, making the actors dependent of a third party, being expensive, and having possible risks for communication bottlenecks.

\section{CONCLUSIONS}

Collaboration is one of the key building blocks for improved business processes and increased profitability within transport chain management. We have suggested an agent-based approach to realize the FREIGHTWISE Framework vision, which is as decentralized as possible while still having the ability to provide means for handling issues related to information security and integrity. However, further work will be carried out within the FREGHTWISE project in order to validate the approach.

\section{REFERENCES}

L.M. Camarinha-Matos, and H. Afsarmanesh. "Elements of a Base VE Infrastructure", Journal of Computers in Industry, vol. 51, no. 2, pp. 139-163, 2003.

L.C. Cardoso, and E. Oliveira, Virtual Enterprise Normative Framework Within Electronic Institutions, Engineering Societies in the Agents World V, Lecture Notes in Artificial Intelligence, Vol. 3451, pp. 14-32, Springer, 2005.

P. Davidsson. "Categories of Artificial Societies", Engineering Societies in the Agents World II, Lecture Notes in Artificial Intelligence, Vol. 2203, Springer, 2001.

P. Davidsson, A. Hederstierna, A. Jacobsson, J.A. Persson, et al., "The Concept and Technology of Plug and Play Business", 8th International Conference on Enterprise Information Systems, 2006.

P. Davidsson and A. Jacobsson. "Towards NormGoverned Behavior in Virtual Enterprises", Intelligent Agents in the Evolution of Web and Applications, Studies in Computational Intelligence, Vol-ume 167, Springer, 2009.

P. Davidsson, L. Ramstedt, and J. Törnquist, "InterOrganization Interoperability in Transport Chains Using Adapters Based on Open Source Freeware", Interoperability of Enterprise Software and Applications, Springer, 2005.

K.E. Fjørtoft, H. Westerheim, M.K. Natvig, J.T. Pedersen, T. Georgios, et al., "WP13 FREIGHTWISE framework architecture, release 2", 2008.

A. Jacobsson, P. Davidsson. "An Analysis of Plug and Play Business Software", Project E-Society: Building Bricks (Proceedings of the Sixth IFIP International Conference on e-Commerce, e-Business and eGovernment), Springer, 2006.

A. Jacobsson, P. Davidsson. "A Formal Analysis of Virtual Enterprise Creation and Operation”, eds. Król, D, and Nguyen, NT: Intelligence Integration in Distributed Knowledge Management, Information Science Reference, 2008.

N.R. Jennings, P. Faratin, A.R. Lomuscio, S. Parsons, C. Sierra, and M. Wooldridge, "Automated Negotiation: Prospects", Methods and Challenges. International 
Journal of Group Decision and Negotiation, vol. 10, no. 2, pp. 199-215, 2001

A. Oram (ed.). Peer-to-Peer: Harnessing the Power of Disruptive Technologies, O'Reilly, 2001.

J.S. Rosenschein, and G. Zlotkin, Rules of Encounter: Designing Conventions for Automated Negotiation among Computers, MIT Press, Cambridge MA, 1994.

R.G. Smith, "The Contract Net Protocol: High-Level

Communication and Control in a Distributed Problem Solver", IEEE Transactions on Computers, Vol. C-29, no. 12, pp. 1104-1113, 1980.

I. Stoica, R. Morris, D. Karger, M.F. Kaashoek, H. Balakrishnan. "Chord: A Scalable Peer-to-peer Lookup Service for Internet Applications", SIGCOMM'01, ACM, 2001.

L.A. Wolsey. Integer Programming, Wiley, 1998. 